\title{
Clinical and laboratory diagnosis of congenital Zika virus syndrome and diaphragmatic unilateral palsy: case report
}

\author{
Alex Sandro Rolland Souza 1 \\ Marli Tenorio Cordeiro 2 \\ Jucile do Amaral Meneses 3 \\ Emanuele Honorato 4 \\ Edward Araujo Júnior 5 \\ Priscila Mayrelle da Silva Castanha 6 \\ Bruna Varginha Ramos Caiado 7 \\ Silvia de Lourdes Loreto Faquini 8 \\ Orlando Gomes Santos Neto 9 \\ Juliana Araújo de Carvalho Schettini 10 \\ Luisa Medeiros de Mello 11 \\ Ana Catarina Ishigami 12 \\ Rafael Dhalia 13 \\ 1,4,8,9 Centro aos cuidados da mulher . Instituto de Medicina Integral Prof. Fernando Figueira (IMIP). Rua dos Coelhos, 300. Boa Vista. Recife, PE, Brazil. CEP: 50.070-550. \\ E-mail: alexrolland@uol.com.br \\ 2,6,7,13 Departamento de Virologia. Centro de Pesquisa Aggeu Magalhães. Fundação Oswaldo Cruz (Fiocruz). Recife, PE, Brasil. \\ 5 Departamento de Obstetrícia. Escola Paulista de Medicina. Universidade Federal de São Paulo (EPM-UNIFESP). São Paulo, SP, Brasil. \\ ${ }^{10}$ Laboratório de Pesquisa Translacional. Instituto de Medicina Integral Prof. Fernando Figueira. Recife, PE, Brasil. \\ 3,11,12 Departamento de Neonatologia. Instituto de Medicina Integral Prof. Fernando Figueira. Recife, PE, Brasil.
}

\begin{abstract}
Introduction: several birth defects associated to congenital Zika virus infection have been reported, although the clinical features have not been fully characterized.

Description: this is the first case report on unilateral diaphragmatic paralysis diagnosed on a neonate with congenital Zika confirmed by the examination of the amniotic fluid through polymerase chain reaction (ZIKV RT-PCR) and the examination of cerebrospinal fluid by serological test (IgM ZIKV-ELISA) after birth. The main manifestations detected by intrauterine ultrasound were: microcephaly, ventriculomegaly, intracranial calcifications, enlarged cisterna magna, increased amniotic fluid index and fetal akinesia syndrome. The newborn had acute respiratory failure in the first hours of life, requiring mechanical ventilation. The X-ray of the chest showed unilateral diaphragmatic paralysis and cardiomegaly.

Discussion: diaphragmatic palsy in congenital Zika has not been previously reported, the etiopathogenic mechanisms of this event in congenital Zika virus needs to be elucidated. Key words Microcephaly, Arbovirus infections, Arthrogryposis, Prenatal ultrasound, Respiratory paralysis
\end{abstract}




\section{Introduction}

Zika virus (ZIKV) was first isolated in the Zika forest in Uganda, Africa in 1947 and thereafter in 1952 the first human with ZIKV infection was identified in Uganda and the United Republic of Tanzania. ZIKV outbreaks were described in 2007 in the Yap State (Micronesia) and in 2013 in the French Polynesia in the South Pacific. ${ }^{1}$ In Brazil, the first report of autochthonous transmission of ZIKV was in 2015.2 In this same year, the Brazilian Ministry of Health announced the increase of neonatal microcephaly cases following a ZIKV outbreak. The causal link between ZIKV and microcephaly strengthened after the identification of ZIKV in the amniotic fluid from two pregnant women positive for ZIKV with fetal abnormalities (by polymerase chain reaction test-RT-PCR) in Paraíba State (Northeast of Brazil) and the detection of ZIKV genome in tissues of a newborn with microcephaly who died after birth. 3

ZIKV is a RNA virus transmitted by the Aedes mosquitos bites to humans and primates. ${ }^{1}$ The less common modes of transmission are through blood transfusion, sexual contact and perinatal transmission. ${ }^{4}$ The possible routes of perinatal transmission are transplacental, during delivery, during breastfeeding and possibly by close contact between mother and newborn after ZIKV was identified in human saliva.4,5 However, the exact mechanism of perinatal transmission remains unknown.

Thereafter, many different congenital neurological malformations such as microcephaly, agyria, hydrocephalus, brain atrophy, brain calcifications in the white matter, corpus callosum and vermiandysgenesis, enlarged cisterna magna, asymmetric hemispheres, unilateral ventriculomegaly and displacement of the midline. Other malformations as fetal akinesia syndrome, asymmetric eyes with cataracts, intraocular calcifications, and/or unilateral macular lesions and intrauterine growth restriction were described. 3,6

Diaphragmatic paralysis is an extremely rare event. Post viral neuropathy and phrenic nerve involvement have been described following several viral infections like herpes zoster, poliovirus, dengue (DENV), West Nile virus and human immunodeficiency virus infections among adults.? Diaphragmatic palsy and cardiomegaly were not to be reported in neonates with congenital Zika. Here we first describe a unilateral diaphragmatic palsy case of a newborn with laboratorial confirmation of congenital Zika. The diagnostic of ZIKV infection was confirmed by testing the amniotic fluid through polymerase chain reaction (ZIKV RT-PCR) and by serological test (IgM ZIKV-ELISA) performed in the cerebrospinal fluid of the neonate.

\section{Description}

A 17-year-old primigravida from Pernambuco (Northeast of Brazil) was referred to the Fetal Medicine Unit at $27^{\text {th }}$ week of gestation was suspected of fetal microcephaly by a previous prenatal ultrasound. The woman had characteristic signs and symptoms of ZIKV infection at $14^{\text {th }}$ weeks of gestation (rash and fever). Laboratorial tests for screening congenital infections, such as TORCH infections [Toxoplasmose, Rubella, Cytomegalovirus (CMV), and Herpes infections and others] were not performed. She took folic acid supplementation and ferrous sulfate and was vaccinated against influenza, diphtheria and tetanus on the $11^{\text {th }}$ week. The patient denied personal or family history of microcephaly or other congenital abnormalities, the consumption of illicit drugs, alcohol, smoking, cosmetics or corticoids, or having any contact with pesticides, insecticides or ionizing radiation.

At 27 week of gestation, the fetal abnormalities on the morphological ultrasound where the biparietal diameter (BPD) was $54 \mathrm{~mm}$ and the head circumference (HC) was $216 \mathrm{~mm}$ (BPD and HC two standard deviations [SD] were below the mean for gestational age) (Figure 1). The abdominal circumference (AC) and femur length (FL) were normal for gestational age. Moreover, it was also observed agenesis of cerebellar vermix and enlarged cisterna magna (measuring $23 \mathrm{~mm}$ ), ventriculomegaly with increased posterior horn of the lateral ventricle (measuring $20 \mathrm{~mm}$ ), periventricular and diffuse calcification in the cerebral hemispheres, forehead flattened and increase in amniotic fluid index (measuring $19 \mathrm{~cm}$ ) (Figure 1). The upper and lower limbs were not adequately assessed because of the inadequate fetal position. Other organs and systems were apparently normal. Doppler velocimetry of the umbilical and uterine arteries were normal.

At 29 weeks of gestation, the intrauterine fetal growth restriction was observed. Prenatal ultrasound monitoring was conducted every 15 days and a higher decrease in the SD of the curve to the mean gestational age of the $\mathrm{HC}$ were observed.

Trans-abdominal amniocentesis guided by ultrasound was performed at 29 weeks of gestation and the amniotic fluid sample was ZIKV positive by quantitative real-time reverse transcription-polymerase chain reaction (qRT-PCR). At 31 weeks of 
gestation, the maternal hematological profile (hemogram) was normal and serologic tests for TORCH (syphilis, toxoplasmosis, rubella, cytomegalovirus, herpes simplex, B19 parvovirus and HIV infection) were negative.

The woman had vaginal delivery of a female newborn at 39 weeks. The newborn's birth weight was 2,565 $\mathrm{g}$ (small for gestational age). The Apgar scores at birth were six and seven in the first and fifth minutes, respectively, and the maneuver resuscitation with positive pressure ventilation and mask were needed. The newborn was referred for treatment at the neonatal intensive care unit (NICU) due to respiratory distress and was placed in a non-invasive ventilation with nasal continuous positive airway pressure and positive end expiratory pressure of +5 , and a fraction of inspired oxygen $\left(\mathrm{FiO}_{2}\right)$ of 0.40 . Intravenous nutrition was initiated.

A complete physical examination, included a careful measurement of the head circumference, length, and weight, was performed by a pediatrician after 24 hours of birth of the newborn's life. The occipitofrontal circumference was $28 \mathrm{~cm}$, more than three SD below the mean for gestational age and gender. The newborn's length was $46 \mathrm{~cm}$ and the thoracic circumference was $30 \mathrm{~cm}$. After birth, microcephaly and other dysmorphic features as fetal akinesia syndrome/arthrogryposis of the hip and hands as well as bilateral clubfoot were found (Figure 2). The brain transfontanellar ultrasound revealed bilateral ventricular enlargement and widespread brain calcifications.

Blood samples from the mother and child were collected to track dengue (DENV), chikungunya (CHIKV) and ZIKV infection. The newborn was also tested for TORCH. CSF was collected from the newborn to assess ZIKV infection through for molecular (ZIKV RT-PCR) and serologic tests (ZIKV IgM-ELISA). Plaquet Reduction Neutralization Test (PRNT) was performed to assess neutralizing antibodies of ZIKV and DENV following a modified protocol previously described. The PRNT results

Figure 1

Fetal microcephaly.

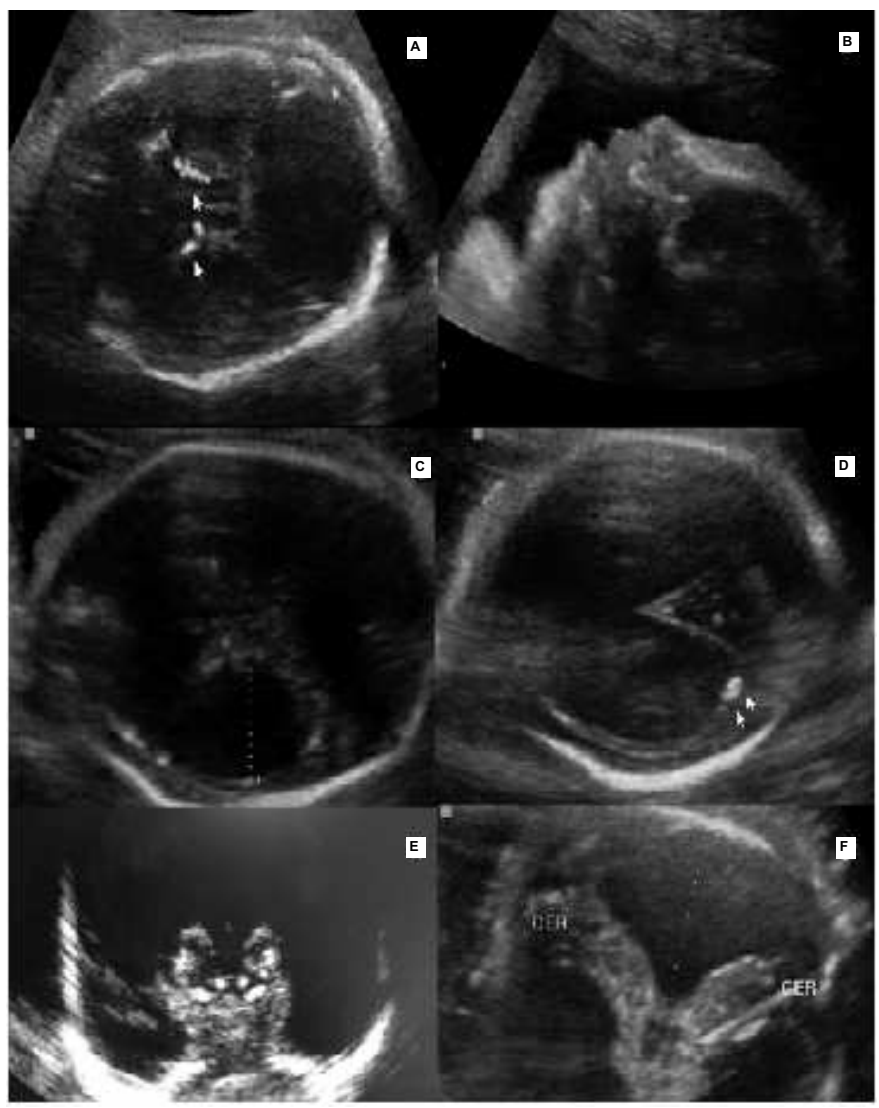

A. Diffuse calcification in the cerebral hemispheres; B. Forehead flattened; C. Ventriculomegaly, periventricular calcification and enlarged cisterna magna; D. Ventriculomegaly and periventricular calcification; E. Brain transfontanellar ultrasound revealed ventriculomegaly and widespread of the brain calcifications; F. Prenatal ultrasound revealed cisterna magna and bilateral ventricular enlargements. 
Figure 2

Microcephaly and other dysmorphic features.

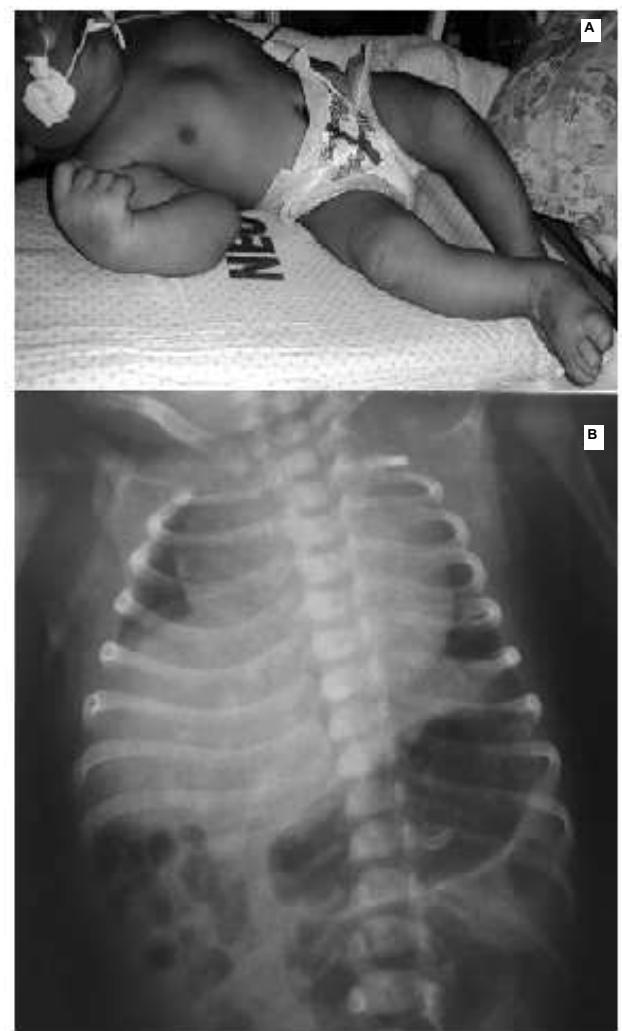

A. Fetal akinesia syndrome/arthrogryposis of the hip and hands and bilateral clubfoot; B. Chest X-ray. Note normal lungs with a unilateral diaphragmatic paralysis and cardiomegaly.

Table 1

\begin{tabular}{|c|c|c|c|c|c|c|c|c|}
\hline \multirow[t]{2}{*}{ Patient } & \multirow[t]{2}{*}{ Sample } & \multicolumn{3}{|c|}{ RT-PCR } & \multicolumn{2}{|c|}{$\lg M(P / N) *$} & \multirow{2}{*}{$\frac{\mathbf{I g G}}{\mathrm{DENV}}$} & \multirow[t]{2}{*}{ Interpretation } \\
\hline & & ZIKV & DENV & CHIKV & ZIKV & DENV** & & \\
\hline Mother & Serum & ND & ND & ND & 1.4 & 2.2 & Positive & \\
\hline \multirow[t]{2}{*}{ Newborn } & CSF & ND & ND & ND & 24.5 & 0.7 & & ZIKV IgM positive \\
\hline & Serum & ND & ND & ND & 13.6 & 0.9 & Positive & ZIKV IgM positive \\
\hline
\end{tabular}

$N D=$ Not Detected; $*(P / N)=$ Ratio OD sample/OD negative control - Values $<2$ are negative; $>3$ are positive; values $2-3$ are considered equivocal; $* *$ DENV $=$ dengue virus $1-4$ mixture.

showed that mother and the neonate had anti-dengue anti-bodies for DENV-3 and DENV-4 in the past, but not for serotypes 1 and 2 (Table 1).

The chest X-ray was performed to evaluate persistent respiratory distress and revealed normal lungs with a unilateral diaphragmatic paralysis and cardiomegaly (Figure 2). The color Doppler echocardiography showed a $2.5 \mathrm{~mm}$ patent ductus arteriosus with a left to right shunt and left cardiac enlargement. The patient was treated with diuretics 
(furosemide) and was still on ventilatory support. The updated chest $\mathrm{x}$-ray was normal except for the unilateral diaphragmatic paralysis. Congenital infections such as syphilis, toxoplasmosis, rubella, cytomegalovirus, and herpes simplex virus were ruled out of the newborn. The neonate is still at the intensive care unit with respiratory support until normal function of the diaphragm which is essential for effective ventilation.

\section{Discussion}

We first describe a case of unilateral diaphragmatic palsy of a newborn with laboratorial confirmation of congenital Zika. In addition, we emphasized the need to perform a chest X-ray on the newborn with respiratory distress to evaluate the possibility of unilateral diaphragmatic paralysis. It is important to highlight the not yet description associating unilateral diaphragmatic paralysis and congenital ZIKV infection.

Diaphragmatic paralysis described in the chronic inflammatory demyelinating polyneuropathy might result to neurological diseases affecting the phrenic nerve, including Guillain-Barré syndrome and in two reports were associated to phrenic neuropathy following DENV infection in adults. ZIKV infection is associated to Guillain-Barré syndrome in adults but there are no reports on newborns. ${ }^{7}$ Unilateral diaphragmatic paralysis has been described by following several viral infections such as herpes zoster, poliovirus, West Nile virus and human immunodeficiency virus. This particular finding has not been reported before in any of the studies published to this present date and the exact mechanism of phrenic neuropathy associated to Zika infection still needs to be elucidated.

The etiopathigenic mechanism involved in the diaphragmatic paralysis in congenital ZIKV infection are not known and further studies are necessary to investigate this specific abnormality.

This case of congenital Zika was discovered in Pernambuco State which is considered the epicenter of congenital infections attributed to ZIKV in Brazil. ${ }^{3}$ The rapid outbreak of ZIKV in Brazil and also in Latin America is probably the result of a recent introduction of the virus in a naïve population, in addition to the environmental factors such as high densities population of Aedes aegyti, the main vector of arboviruses worldwide. 8

The identification of ZIKV is still a challenge, especially during the pregnancy, of fetal brain neurodevelopment disorders and maternal symptoms. The amniocentesis was a fundamental impor- tance to confirm the diagnosis. Amniocentesis is a low-risk invasive procedure and was performed successfully. ${ }^{3}$ We recommend amniocentesis to be performed in the presence of fetal abnormalities suggestive for ZIKV infection because the amniotic fluid is the only place to identify ZIKV by RT-PCR in the uterus, particularly in advanced gestational age confirming the diagnosis of fetal infection.

ZIKV genome was detected in the amniotic fluid at 29 weeks of gestation by RT-PCR. However, ZIKV RNA was not detected after birth (both the mother and neonate serum samples) probably because the acute infection occurred at early gestation. ZIKV IgM in the mother's serum sample test was negative. However, specific ZIKV IgM antibodies were detected in the neonate's CSF and serum sample test. IgM does not cross the placenta or the blood-brain barrier, the finding of IgM in the CSF indicates that the newborn had ZIKV infection in the CNS. In addition, the finding of IgM anti-bodies in the neonate serum sample indicated that the patient had the infection in the uterus. It is not yet known for how long these ZIKV IgM anti-bodies will be detected in the CSF and in the blood.

The PRNT results showed that the mother and the neonate had anti-dengue anti-bodies for DENV-3 and DENV-4 in the past, but not for serotypes 1 and 2 , suggesting that the maternal $\mathrm{IgG}$ anti-bodies transplacental passed to the newborn. Both mother and neonate presented high titter of neutralizing antibodies for ZIKV in the serum, although, the newborn was higher (anti-bodies produced by the mother and newborn), almost the double. Importantly, neutralizing anti-bodies for ZIKA virus were also detected in the CSF by confirming the findings of $\operatorname{IgM}$ in the CSF. It is noteworthy that IgM antibodies do not cross the placental barrier and this result does not suggest the infection is associated to ZIKV and DENV.

Most reports associate ZIKV and microcephaly when the pregnancy is infected in the first quarter however, in this case, the woman reported symptoms suggestive to ZIKV infection at 14 weeks of gestation in the beginning of the second quarter of gestation. Although, a study suggests that fetal impairment could occur at any time during the pregnancy. ${ }^{9}$ The patient did not perform tests for screening congenital infections at the time of symptomatology. Microcephaly diagnosis was performed prenatally. During the gestation was also observed that the growth of the fetal head decreased, suggesting that there is a stop of the growth. The measurement of head circumference through ultrasound shows a small variability, both inter-observer and intra- 
observer. A transfontanellar brain ultrasound was used to confirm microcephaly of the newborn. ${ }^{10} \mathrm{~A}$ cerebral computerized tomography scan was not performed because the acoustic window of the anterior fontanelle was sufficient for the newborn brain examination be performed by transfontanellar ultrasound.

Microcephaly associated to intracranial calcification and ventriculomegaly is described in the literature as suggestive to other congenital infections, ${ }^{9}$ but the association of these manifestations with agenesis of the cerebellar vermix and arthrogryposis still needs scientific proof. There are several other causes of microcephaly described in the literature [genetics, hypoxic-ischemic, vascular disorders, systemic and metabolic disorders, drug exposure during pregnancy (alcohol and chemicals), severe malnutrition during pregnancy (intrauterine malnutrition), and maternal phenylketonuria]. 5

All morphological alterations observed prenatally were confirmed, adding to other diagnoses, such as the arthogryposis which is the presence of congenital joint contractures involving at least two different body areas both the upper and lower limbs. It is a descriptive diagnosis present in many diseases with varying etiologies and is a known observer in the presence of ZIKV infection. We do not know the exact mechanism of arthrogryposis. If it is by direct action of the virus in the articulations, aberrant activation of the immune response, decreased fetal

\section{References}

1. Cao-Lormeau VM, Roche C, Teissier A, Robin E, Berry AL, Mallet HP, Sall AA, Musso D. Zika virus, French Polynesia, South Pacific, 2013. Emerg Infect Dis. 2014; 20: $1085-6$

2. Zanluca C, Melo VC, Mosimann AL, Dos Santos GI, Dos Santos CN, Luz K. First report of autochthonous transmission of Zika virus in Brazil. Mem Inst Oswaldo Cruz. 2015; 110: 569-72

3. Oliveira Melo AS, Malinger G, Ximenes R, Szejnfeld PO, Alves Sampaio S, Bispo de Filippis AM. Zika virus intrauterine infection causes fetal brain abnormality and microcephaly: tip of the iceberg? Ultrasound Obstet Gynecol. 2016; 47 (1): 6-7.

4. Karwowski MP, Nelson JM, Staples JE, Fischer M, Fleming-Dutra KE, Villanueva J, Powers AM, Mead P, Honein MA, Moore CA, Rasmussen SA. Zika virus disease: A CDC Update for Pediatric Health Care Providers.Pediatrics. 2016; 137 (5). Disponível em http://pediatrics.aappublications.org/content/pediatrics/earl y/2016/03/22/peds.2016-0621.full.pdf movement in the uterus determining the joint involvement, and deformities found, or by other causes.

This report is a description of a laboratorial confirmed congenital ZIKV syndrome in the gestational period. The neonate presented respiratory distress after birth that was diagnosed as unilateral diaphragmatic paralysis according to the chest Xray. Based on this finding, we suggest performing chest X-ray in neonates suspected with congenital Zika to assess the presence of diaphragmatic palsy. In addition, the neonate is still at the intensive care unit with respiratory support until there is uncoordinated activity of the diaphragm, an essential muscle for effective ventilation.

There are some questions whereas answers are to be investigated in further studies: Why this woman's newborn was affected by the ZIKV once she was in her $14^{\text {th }}$ weeks of gestation, when only a few women had newborns affected and most of the infections were in the first quarter? What is the mechanism of the unilateral diaphragmatic palsy in newborns with congenital ZIKV?

\section{Acknowledgments}

Authors would like to thank the pregnant woman.

5. Pan American Health Organization. Epidemiological Alert: Neurological syndrome, congenital malformations, and Zikavirus infection. Implicantion for public health in americas. Washington, DC: World Health Organization, Pan American Health Organization; December 1, 2015. [access Jan 29, 2016]. Available at: http://paho.org/hq/index/php? option=com_docman\&task=doc_download\&I-temid=\&gid $=32405 \&$ lang $=$ en

6. Marrs C, Olson G, Saade G, Hankins G, Wen T, Patel J, Weaver S. Zikavirus and pregnancy: areview of the literature and clinical considerations (Podcast). Am J Perinatol. 2016; 33 (7): 625-39.

7. Ansari M K, Jha S, Nath A. Unilateral diaphragmatic paralysis following dengue infection. Neurol India. 2010; 58:596-8.

8. Castanha PMS, Cordeiro MT, Martelli CMT, Souza WV, Marques ET, Braga C. Force of infection of dengue serotypes in a population-based study in the northeast of Brazil. Epidemiol Infect. 2012; 141: 1080-8. 
9. Chang C, Ortiz K, Ansari A, Gershwin ME. The Zika outbreak of the 21 st century. J Autoimmun. 2016; 68: 1-13.

10. Miranda-Filho DB, Martelli CM, Ximenes RA, Araújo TV, Rocha MA, Ramos RC, Dhalia R, França RF, Marques Júnior ET, Rodrigues LC. Initial description of the presumed congenital Zikasyndrome. Am J Public Health. 2016; 106 (4): 598-600.

Received on July 6, 2016

Final version presented on October 11, 2016

Approved on October 20, 2016 